FTUV/97-5

hep-ph/9702231 IFIC $/ 97-5$

\title{
Neutrinos and Physics Beyond the Standard Model
}

\author{
J. W. F. Valle \\ Instituto de Física Corpuscular - C.S.I.C. \\ Departament de Física Teòrica, Universitat de València \\ 46100 Burjassot, València, Spain
}

\begin{abstract}
A brief sketch is made of the present observational status of neutrino physics, with emphasis on the hints that follow from solar and atmospheric neutrino observations, as well as cosmological data on the amplitude of primordial density fluctuations. I also briefly review the ways to account for the observed anomalies and some of their implications.
\end{abstract}




\title{
Neutrinos and Physics Beyond the Standard Model
}

\author{
J. W. F. Valle ${ }^{\mathrm{a} *}$ \\ anstituto de Física Corpuscular - C.S.I.C. \\ Departament de Física Teòrica, Universitat de València \\ 46100 Burjassot, València, Spain
}

A brief sketch is made of the present observational status of neutrino physics, with emphasis on the hints that follow from solar and atmospheric neutrino observations, as well as cosmological data on the amplitude of primordial density fluctuations. I also briefly review the ways to account for the observed anomalies and some of their implications.

\section{INTRODUCTION}

Two puzzles exist associated to neutrinos: if massless, they would be only fermions with this property. If massive, why are their masses so much smaller than those of their charged brothers? The fact that neutrinos are the only electrically neutral elementary fermions may hold the key to the answer, namely neutrinos could be Majorana fermions, in some sense the most fundamental ones. In this case the supression of their mass could be associated to lepton number conservation. But so far we do not know.

It is beyond any doubt that, one of the most fundamental drawbacks of the Standard Model is the fact that it says so little about the properties of neutrinos. Their masslessness is not dictated by a fundamental underlying principle, such as gauge invariance in the case of the photon. Most extensions of the Standard Model require neutrinos to be massive. One interesting aspect of many models where neutrinos have non-vanishing masses is that they lead to effects that could be experimentally tested, even outside the conventional realm of neutrino experiments, such as underground experiments. In some cases one may probe, though indirectly, the underlying physics at high energy accelerators.

\footnotetext{
*Supported by DGICYT grant PB95-1077 and in part by EEC under the TMR contract ERBFMRX-CT96-0090. I thank the organizers for the hospitality, and H. Nunokawa and F. Vissani for helping me prepare the manuscript and M. A. Diaz for proof-reading it.
}

From the observational point of view nonzero neutrino masses now seem required in order to account for the data on solar and atmospheric neutrinos, as well as the (hot) dark matter in the universe [1]. But before overviewing the present observational limits and hints in favour of massive neutrinos, let us make a few general remarks about the theoretical models [2].

\section{MODELS}

One of the simplest extensions of the electroweak theory consists in adding isosinglet neutral heavy leptons (NHLS), such as right handed neutrinos, as in the seesaw model [3]. In this case the NHLS have a large Majorana mass term $M_{R}$, which violates total lepton number, or B-L (baryon minus lepton number), a symmetry that plays an important role in many extended gauge models 4 . The masses of the light neutrinos are obtained by diagonalizing the following mass matrix

\begin{tabular}{c|cc} 
& $\nu$ & $\nu^{c}$ \\
\hline$\nu$ & 0 & $D$ \\
$\nu^{c}$ & $D^{T}$ & $M_{R}$
\end{tabular}

where $D=h_{D}\langle H\rangle / \sqrt{2}$ is the Dirac mass matrix and $M_{R}=M_{R}^{T}$ is the isosinglet Majorana mass. In the seesaw approximation, one finds

$M_{L}=-D M_{R}^{-1} D^{T}$. 
This mechanism is able to explain naturally the relative smallness of neutrino masses [3]. Although the seesaw idea was suggested in the context of $\mathrm{SO}(10)$ or left-right symmetric extensions where lepton number is a part of the gauge symmetry [ [4], it may be directly introduced in the Standard Model. Though it is natural to expect $M_{R}$ to be large, one can not make any firm guess, as its magnitude heavily depends on the model. As a result one can not make any real prediction for the corresponding light neutrino masses that are generated through this so-called seesaw mechanism [3].

Although attractive, the seesaw mechanism is by no means the only way to generate neutrino masses. There is a large diversity of possible schemes to generate neutrino masses, which do not require any new large mass scale. For example, it is possible to start from an extension of the lepton sector of the $S U(2) \otimes U(1)$ theory by adding a set of two 2-component isosinglet neutral fermions, denoted $\nu^{c}{ }_{i}$ and $S_{i}$. In this case there is an exact L symmetry that keeps neutrinos strictly massless, as in the Standard Model. The conservation of total lepton number leads to the following form for the neutral mass matrix

\begin{tabular}{c|ccc} 
& $\nu$ & $\nu^{c}$ & $S$ \\
\hline$\nu$ & 0 & $D$ & 0 \\
$\nu^{c}$ & $D^{T}$ & 0 & $M$ \\
$S$ & 0 & $M^{T}$ & 0
\end{tabular}

This form has also been suggested in various theoretical models [5], including many of the superstring inspired models. In the latter case the zeros of eq. (3) naturally arise due to the absence of Higgs fields to provide the usual Majorana mass terms, needed in the seesaw model [6]. The implications of eq. (3) are interesting on their own right, and the model represents a conceptually simple and phenomenologically rich extension of the Standard Model, which brings in the possibility that a wide range of new phenomena be sizeable. These have to do with neutrino mixing, universality, flavour and $\mathrm{CP}$ violation in the lepton sector [7,8], as well as direct effects associated with the NHL production in $Z$ decays [9]. A remarkable feature of this model is the possibility of non-trivial neutrino mixing despite the fact that neutrinos are strictly massless. This treelevel effect was exploited in ref. [10,11. Moreover, there are loop-induced lepton flavour and $\mathrm{CP}$ non-conservation effects whose rates are precisely calculable [7,8,12]. I repeat that this is remarkable due to the fact that physical light neutrinos are massless, as in the Standard Model. This feature is the same as what happens in the supersymmetric mechanism of flavour violation [13]. Indeed, in the simplest case of SU(5) supergravity unification, there are flavour violating processes, like $\mu \rightarrow e \gamma$, despite the fact that in $\mathrm{SU}(5)$ neutrinos are protected by B-L and remain massless. The supersymmetric mechanism and that of eq. (3) differ in that the lepton flavour violating (LFV) processes are induced in one case by NHL loops, while in supersymmetry they are induced by scalar boson loops. In both cases the particles in the loops have masses at the weak scale, leading to potentially sizeable rates. The allowed LFV branching ratios in supersymmetry have been discussed here by Barbieri [14,15]. In the NHL model, as a result of the masslessness of the physical neutrinos, the allowed value for the LFV branching ratios is also maximized in the class of neutrino mixing models. Indeed, one can show that some of the LFV rates can be sizeable enough to be experimentally detectable [16 18].

Clearly, one can easily introduce non-zero masses in this model through a $\mu S S$ term that could be proportional to the VEV of a singlet field $\sigma$ 19. In contrast to the seesaw scheme, the neutrino masses are directly proportional to $\langle\sigma\rangle$, a fact which is very important for the phenomenology of the Higgs boson sector.

There is also a large variety of possible radiative schemes to generate neutrino masses. The prototype models of this type are the Zee model and the model suggested by Babu [20]. In these models lepton number is explicitly broken, but it is easy to realize them with spontaneous breaking of lepton number. For example in the version suggested in ref. 21] the neutrino mass arises from the diagram shown in Fig. 1 These models do not require one to introduce a large mass scale. It is quite possible to embed such schemes so as to 


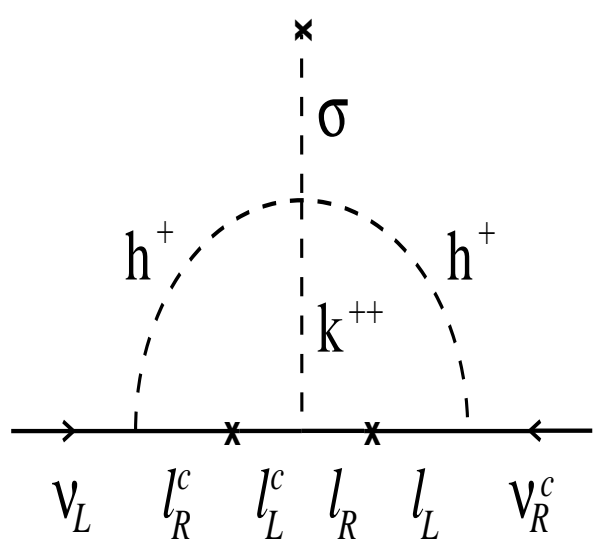

Figure 1. Two-loop-induced Neutrino Mass.

have the spontaneous violation of the global lepton number symmetry. In these models again the neutrino masses are directly proportional to $\langle\sigma\rangle$ or some positive power of that, depending on the lepton numbers assignments of the model. Thus the scale at which such a symmetry gets broken does not need to be high, as in the original proposal [22], but can be rather low, close to the weak scale 23. Such models are very attractive and lead to a richer phenomenology, as the extra particles required have masses at scales that could be accessible to present experiments.

\section{LIMITS ON NEUTRINO MASSES AND MIXINGS}

\subsection{Laboratory Limits}

The best limits on the neutrino masses can be summarized as [24]:

$m_{\nu_{e}} \lesssim 5 \mathrm{eV}, \quad \mathrm{m}_{\nu_{\mu}} \lesssim 170 \mathrm{keV}, \quad \mathrm{m}_{\nu_{\tau}} \lesssim 23 \mathrm{MeV}(4)$

These are the most model-independent of the laboratory limits on neutrino mass, as they follow purely from kinematics. The limits on the $\nu_{e}$ mass comes from beta decay, that on the $\nu_{\mu}$ mass comes from PSI (90 \% C.L.) [25], with further improvement limited by the uncertainty in the $\pi^{-}$mass. On the other hand, the best $\nu_{\tau}$ mass limit now comes from high energy LEP experiment ALEPH [26] and may be substantially improved at a future tau-charm factory 27]. In connection with tritium beta decay limit [28] it is interesting to remark two features in the spectrum. One is an excess of events near the end point of the differential spectrum, at $Q-E_{e} \lesssim 10$ $\mathrm{eV}$, leading to a negative $m^{2}$ value in the fit and probably of instrumental origin. The second is an excess of events at lower electron energies $Q-E_{e} \gtrsim 200 \mathrm{eV}$, also observed by the Mainz group. One possible explanation of this anomaly is the existence of a neutrino with mass $m \sim 200 \mathrm{eV}$ and mixing with the $\nu_{e}$ with a probability $P \sim 1-2 \%$. It has been noted that this is precisely in the range implied by pulsar velocities [1].

In addition, there are limits on neutrino masses that follow from the non-observation of neutrino oscillations. The most stringent bounds come from reactor oscillation experiments [29] $\left(\bar{\nu}_{e}-\nu_{x}\right.$ oscillations); from meson factory oscillation experiments (KARMEN [30], LSND [31]) and from accelerator experiments E531 $\left(\nu_{\mu}-\nu_{\tau}\right)$ 32] and E776 $\left(\nu_{\mu}-\nu_{\tau}\right)$ [33]. The $90 \%$ confidence level (C.L.) exclusion contours of neutrino oscillation parameters in the 2-flavour approximation are summarized in Fig. 2, taken from ref. [34]. Improvements are expected from the ongoing CHORUS and NOMAD experiments at CERN, with a similar proposal at Fermilab 35]. As we have heard at this conference, there are also good prospects for substantial progress at future longbaseline experiments using KEK, CERN and Fermilab neutrino beams aimed at the Kamiokande, Gran Sasso and Soudan underground facilities, respectively. For recent theoretical studies on the possibility of performing $\mathrm{CP}$ violation studies in long-baseline neutrino oscillation experiments see ref. [36].

If neutrinos are of Majorana type a new form of nuclear double beta decay would take place in which no neutrinos are emitted in the final state, i.e. the process by which an $(A, Z-2)$ nucleus decays to $(A, Z)+2 e^{-}$. In such process one would have a virtual exchange of Majorana neutrinos. Unlike the ordinary double beta decay process, the neutrino-less process violates lepton number and its existence would indicate the Majorana na- 


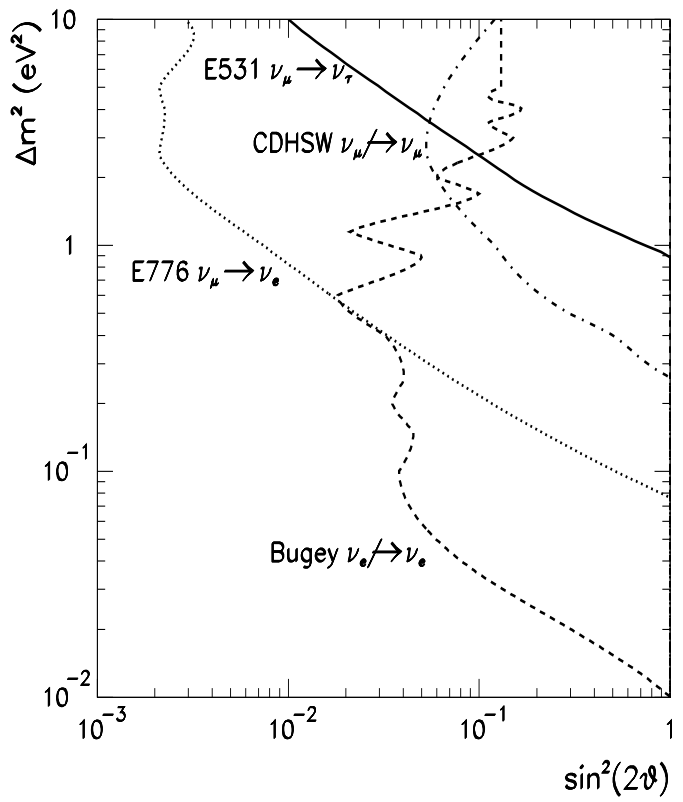

Figure 2. Limits on oscillation parameters.

ture of neutrinos. Because of the phase space advantage, this process is a very sensitive tool to probe into the nature of neutrinos. Present data place an important limit on a weighted average neutrino mass parameter $\langle m\rangle$ 37

$\langle m\rangle \lesssim 1-2 \mathrm{eV}$

depending to some extent on the relevant nuclear matrix elements characterising this process 38. This bound comes from the HeidelbergMoscow experiment and there might be further improvements, also from the IGEX experiment. Note that the parameter $\langle m\rangle$ involves both neutrino masses and mixings. Thus, although rather stringent, this limit in eq. (5) may allow relatively large neutrino masses, as there may be strong cancellations between different neutrino types. This may happen automatically in the presence of suitable symmetries. For example, the decay vanishes if the intermediate neutrinos are Dirac-type, as a result of the corresponding lepton number symmetry [39].

Neutrino-less double beta decay has a great conceptual importance. It has been shown 40] that in a gauge theory of the weak interactions a non-vanishing $\beta \beta_{0 \nu}$ decay rate requires neutrinos to be Majorana particles, irrespective of which mechanism induces it. This is important since in a gauge theory neutrino-less double beta decay may be induced in other ways.

\subsection{Limits from Cosmology}

There are a variety of cosmological arguments that give information on neutrino parameters. In what follows I briefly consider the critical density and the primordial Nucleosynthesis arguments.

\subsubsection{The Cosmological Density Limit}

The oldest cosmological bound on neutrino masses is the one that follows from avoiding the overabundance of relic neutrinos 41]

$m_{\nu_{\tau}} \lesssim 92 \Omega_{\nu} h^{2} \mathrm{eV}$,

where $\Omega_{\nu} h^{2} \leq 1$ and the mass is assumed to be less than $O(1 \mathrm{MeV})$. Here $\Omega_{\nu}=\rho_{\nu} / \rho_{c}$, where $\rho_{\nu}$ is the neutrino contribution to the total density and $\rho_{c}$ is the critical density. The factor $h^{2}$ measures the uncertainty in the determination of the present value of the Hubble parameter, $0.4 \leq h \leq 1$. The factor $\Omega_{\nu} h^{2}$ is known to be smaller than 1 .

For the $\nu_{\mu}$ and $\nu_{\tau}$ this bound is much more stringent than the corresponding laboratory limits eq. (4).

Recently there has been a lot of work on the possibility of an MeV tau neutrino [42]. Such range seems to be an interesting one from the point of view of structure formation 42]. Moreover, it is theoretically viable as the constraint in eq. (6) holds only if neutrinos are stable on the relevant cosmological time scales. In models with spontaneous violation of total lepton number [22] there are new interactions of neutrinos with the majorons which may cause neutrinos to decay into a lighter neutrino plus a majoron, for example [2],

$\nu_{\tau} \rightarrow \nu_{\mu}+J$.

or have sizeable annihilations to these majorons,

$\nu_{\tau}+\nu_{\tau} \rightarrow J+J$. 
The possible existence of fast decay and/or annihilation channels could eliminate relic neutrinos and therefore allow them to be heavier than eq. (6). The cosmological density constraint on neutrino decay lifetime (for neutrinos lighter than 1 $\mathrm{MeV}$ or so) may be written as

$\tau \lesssim 1.5 \times 10^{7}\left(\mathrm{KeV} / m_{\nu_{\tau}}\right)^{2} \mathrm{yr}$,

and follows from demanding an adequate red-shift of the heavy neutrino decay products. For neutrinos heavier than $\sim 1 \mathrm{MeV}$, such as possible for the case of $\nu_{\tau}$, the cosmological limit on the lifetime is less stringent than given in eq. (9).

As we already mentioned the possible existence of non-standard interactions of neutrinos due to their couplings to the Majoron brings in the possibility of fast invisible neutrino decays with Majoron emission [2]. These 2-body decays can be much faster than the visible decays, such as radiative decays of the type $\nu^{\prime} \rightarrow \nu+\gamma$. As a result the Majoron decays are almost unconstrained by astrophysics and cosmology. For a more detailed discussion see ref. 41].

A general method to determine the Majoron emission decay rates of neutrinos was first given in ref. 43]. The resulting decay rates are rather subtle 433 and model dependent and will not be discussed here. The reader may consult ref. [44.2]. The conclusion is that there are many ways to make neutrinos sufficiently short-lived that all mass values consistent with laboratory experiments are cosmologically acceptable. For neutrino decay lifetime estimates see ref. [2, 44, 45].

\subsubsection{The Nucleosynthesis Limit}

There are stronger limits on neutrino lifetimes and/or annihilation cross sections arising from cosmological nucleosynthesis considerations. Recent contradictory data on the primordial deuterium abundance 46, 47 have stimulated a lot of work on the subject 4480. If massive $\nu_{\tau}$ 's are stable on the nucleosynthesis time scale, $\left(\nu_{\tau}\right.$ lifetime longer than $\sim 100 \mathrm{sec}$ ), they can lead to an excessive amount of primordial helium due to their large contribution to the total energy density. This bound can be expressed through an effective number of massless neutrino species $\left(N_{\nu}\right)$.
Using $N_{\nu}<3.4-3.6$, the following range of $\nu_{\tau}$ mass has been ruled out 51,52

$0.5 \mathrm{MeV}<m_{\nu_{\tau}}<35 \mathrm{MeV}$

If the nucleosynthesis limit is taken less stringent the limit loosens somewhat. However it has recently been argued that non-equilibrium effects from the light neutrinos arising from the annihilations of the heavy $\nu_{\tau}$ 's make the constraint stronger and forbids all $\nu_{\tau}$ masses on the few $\mathrm{MeV}$ range.

One can show that if the $\nu_{\tau}$ is unstable during nucleosynthesis [53] the bound on its mass is substantially weakened translated as a function of the assumed lifetime [53].

Even more important is the effect of neutrino annihilations [54]. Fig. 2 gives the effective number of massless neutrinos equivalent to the contribution of massive neutrinos with different values of the coupling $g$ between $\nu_{\tau}$ 's and $J$ 's, expressed in units of $10^{-5}$. For comparison, the dashed line corresponds to the Standard Model $g=0$ case. One sees that for a fixed $N_{\nu}^{\max }$, a wide range of tau neutrino masses is allowed for large enough values of $g$. No $\nu_{\tau}$ masses below $23 \mathrm{MeV}$ can be ruled out, as long as $g$ exceeds a few times $10^{-4}$. One can express the above results in the $m_{\nu_{\tau}}-g$ plane, as shown in Fig. 1 . The region above each curve is allowed for the corresponding $N_{e q}^{\max }$. One sees that the constraints on the mass of a Majorana $\nu_{\tau}$ from primordial nucleosynthesis can be substantially relaxed if annihilations $\nu_{\tau} \bar{\nu}_{\tau} \leftrightarrow J J$ are present. It is instructive to notice that the required values of $g\left(m_{\nu_{\tau}}\right)$ are reasonable in many majoron models [2,55,54].

\subsection{Limits from Astrophysics}

There are a variety of limits on neutrino parameters that follow from astrophysics, e.g. from the supernova 1987A observations by the Kamiokande and IMB collaborations, as well as from the theory of supernovae, including supernova dynamics [56] and from nucleosynthesis in supernovae [57]. Here I briefly discuss a recent illustration of how supernova physics constrains neutrino parameters 11]. It has been noted a long time ago that, in some circumstances, massless 


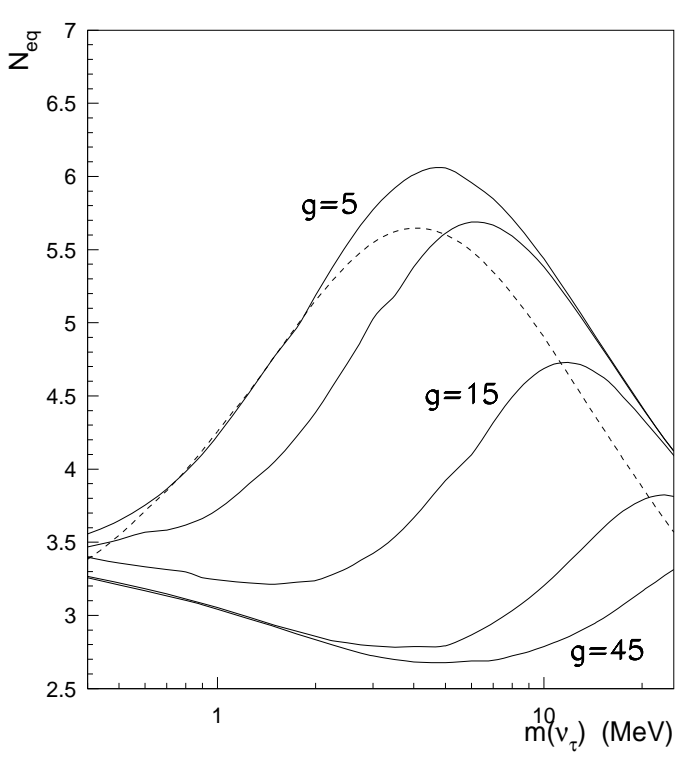

Figure 3. Contribution of a heavy $\nu_{\tau}$ to nucleosynthesis in terms of the equivalent number of massless neutrinos.

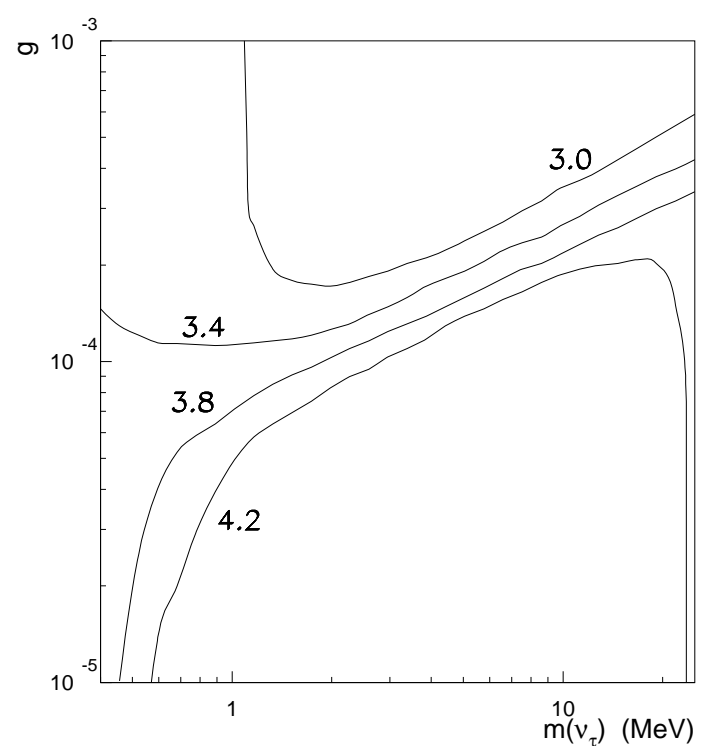

Figure 4. The values of $g\left(m_{\nu_{\tau}}\right)$ above each line would be allowed by nucleosynthesis if one adopts the $N_{e q}^{\max }=3,3.4,3.8,4.2$ (from top to bottom).

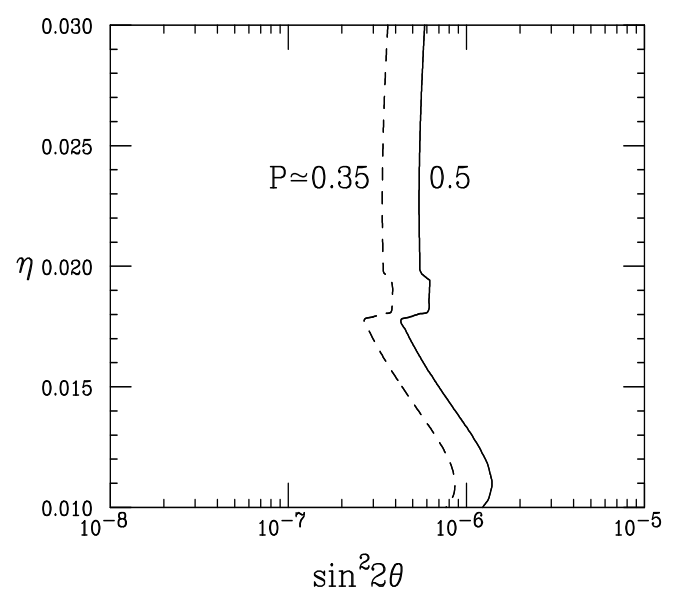

Figure 5. Constraints on massless neutrino mixing from SN1987A.

neutrinos may be mixed in the leptonic charged current [10]. Conventional neutrino oscillation searches in vacuo are insensitive to this mixing. However, in such neutrinos may resonantly convert in the dense medium of a supernova 110,11. One can show how the observation of the energy spectrum of the SN1987A $\bar{\nu}_{e}$ 's $[58$ and the $r$ process nucleosynthesis in the supernova [57 may be used to provide very stringent constraints on massless neutrino mixing angles, as illustrated in Fig. 5 and Fig. 6, respectively. The regions to the right of the solid curves are forbidden, those to the left are allowed. For detailed explanation see ref. [11].

As we saw above despite all the limits from laboratory experiments, both at accelerators and reactors, as well as the limits from cosmology and astrophysics, there is considerable room for interesting new effects in the neutrino sector. In addition to searches for neutrino-less double beta decay and neutrino oscillations, specially at long baseline experiments, it is worthwhile to continue the efforts to improve present laboratory limits on neutrino mass. One method sensitive to large masses is to search for distortions in the energy spectra of leptons coming from $\pi, K$ weak decays such as $\pi, K \rightarrow e \nu, \pi, K \rightarrow \mu \nu$, as well as kinks in nuclear $\beta$ decays. 


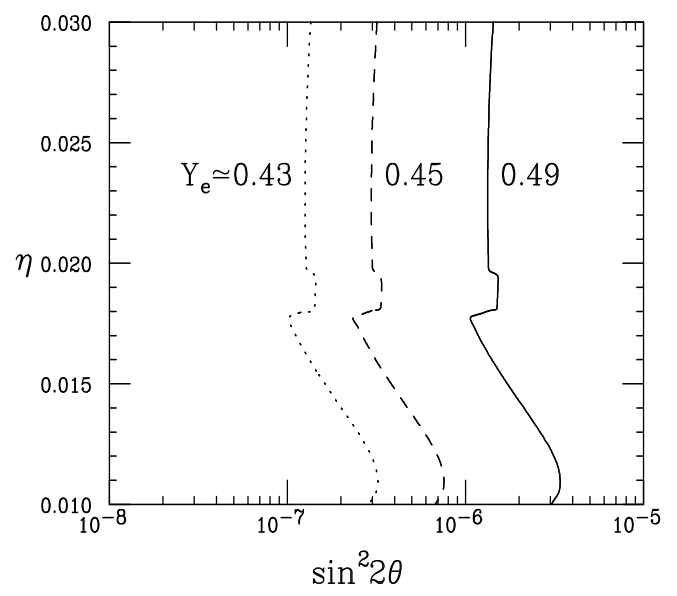

Figure 6. Constraints on massless neutrino mixing arising from nucleosynthesis in supernovae.

\section{HINTS FOR NEUTRINO MASSES}

Detecting nonzero neutrino masses could be very far reaching for the understanding of fundamental issues in particle physics, astrophysics, as well as the large scale structure of our universe. So far the only indications in favour of nonzero neutrino rest masses have been provided by astrophysical and cosmological observations, with a varying degree of theoretical input. We now turn to these.

\subsection{Dark Matter}

Considerations based on structure formation in the Universe have become a popular way to argue in favour of the need of a massive neutrino [59]. Indeed, by combining the observations of cosmic background temperature anisotropies on large scales performed by the COBE satellite 60] with cluster-cluster correlation data e.g. from IRAS [61] one finds that it is not possible to fit well the data on all scales within the framework of the simplest cold dark matter (CDM) model. The simplest way to obtain a good fit is to postulate that there is a mixture of cold and hot components, consisting of about $70 \% \mathrm{CDM}$ with about $25 \%$ hot dark matter (HDM) and a small amount in baryons [59]. The best candidate for the hot dark matter component is a massive neutrino in the few $\mathrm{eV}$ mass range. It has been argued that this could be the tau neutrino, in which case one might expect the existence of $\nu_{e} \rightarrow \nu_{\tau}$ or $\nu_{\mu} \rightarrow$ $\nu_{\tau}$ oscillations. Searches for these oscillations are now underway at CERN, with a similar proposal also at Fermilab [35]. This mass scale is also consistent with the hints in favour of neutrino oscillations reported by the LSND experiment [31].

\subsection{Solar Neutrinos}

So far the averaged data collected by the chlorine [62], Kamiokande [63], as well as by the lowenergy data on pp neutrinos from the GALLEX and SAGE experiments [64,65] still pose a persisting puzzle. The most recent data can be summarised as:

$$
\begin{array}{r}
R_{C l}^{e x p}=(2.55 \pm 0.25) \mathrm{SNU} \\
R_{G a}^{e x p}=(74 \pm 8) \mathrm{SNU} \\
R_{K a}^{e x p}=(0.44 \pm 0.06) R_{K a}^{B P 95}
\end{array}
$$

where $R_{K a}^{B P 95}$ is the BP95 standard solar model (SSM) prediction of ref. [66]. For the gallium result we have taken the average of the GALLEX [64] and the SAGE measurements [65].

Comparing the data of gallium experiments with the Kamiokande data one sees the need for a reduction of the ${ }^{7} \mathrm{Be}$ flux relative to standard solar model [66] expectations. Inclusion of the Homestake data only sharpens the discrepancy, suggesting that the solar neutrino problem is indeed a real problem. The totality of the data strongly suggests that the simplest astrophysical solutions are ruled out, and that new physics is needed [67]. The most attractive possibility is to assume the existence of neutrino conversions involving very small neutrino masses. In the framework of the MSW effect [68] the required solar neutrino parameters $\Delta m^{2}$ and $\sin ^{2} 2 \theta$ are determined through a $\chi^{2}$ fit of the experimental data 2. Fig. 3, taken from ref. [70], shows the $90 \%$ C.L. areas for the in the BP95 model for the case of active neutrino conversions. The fit favours the small mixing solution over the large mixing one, due mostly to the larger reduction of the

${ }^{2}$ For simplicity we neglect theoretical uncertainties, earth effects, as well as details of the neutrino production region. 


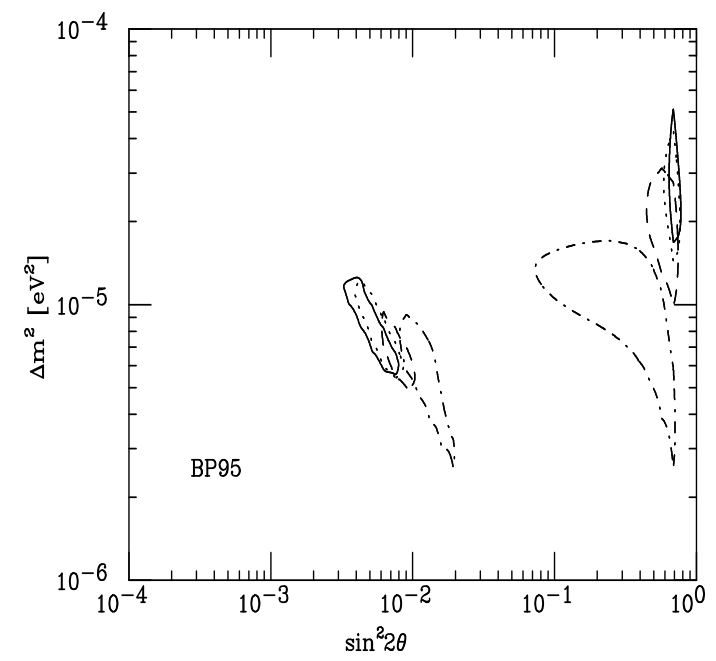

Figure 7. Allowed solar neutrino oscillation parameters for active neutrino conversions.

${ }^{7} \mathrm{Be}$ flux found in the former. Here the different regions in dashed and dot-dashed are associated with a non-zero level of random fluctuations fluctuations in the solar matter density [69]. The existence of such noise-type fluctuations is not excluded by the SSM nor by present helioseismology studies. The solid curves are for the standard case of zero matter density random fluctuations, $\xi=\delta \rho / \rho=0$, corresponding to a smooth solar density profile. The regions inside the other curves correspond to the case where matter density fluctuations are assumed. Noise causes a slight shift of $\Delta m^{2}$ towards lower values and a larger shift of $\sin ^{2} 2 \theta$ towards larger values. The corresponding allowed $\Delta m^{2}$ range for $\xi=8 \%$ is $2.5 \times 10^{-6}<\Delta m^{2}<9 \times 10^{-6} \mathrm{eV}^{2}$ instead of $5 \times 10^{-6}<\Delta m^{2}<1.2 \times 10^{-5} \mathrm{eV}^{2}$ in the noiseless case. The large mixing area is less stable, with a tendency to shift towards smaller $\Delta m^{2}$ and $\sin ^{2} 2 \theta$ values.

It is interesting to note that the ${ }^{7} \mathrm{Be}$ neutrinos are the solar neutrino spectrum component which is most affected by the matter noise. Therefore the Borexino experiment should be an ideal tool for studying the solar matter fluctuations, if sufficiently small errors can be achieved. Its potential in "testing" the level of solar matter density fluctuations is discussed in ref. [70], which also contains a discussion of sterile solar neutrino conversions, as well as a comparison with other solar models.

\subsection{Atmospheric Neutrinos}

The Kamiokande and IMB underground experiments, and possibly also Soudan2, have indications which support an apparent deficit in the expected flux of atmospheric $\nu_{\mu}$ 's relative to that of $\nu_{e}$ 's that would be produced from conventional decays of $\pi$ 's, $K$ 's as well as secondary muon decays 171. Although the predicted absolute fluxes of neutrinos produced by cosmic-ray interactions in the atmosphere are uncertain at the $20 \%$ level, their ratios are expected to be accurate to within $5 \%$. While some of the experiments, such as Frejus and NUSEX, have not found a firm evidence, it has been argued that there may be a strong hint for an atmospheric neutrino deficit that could be ascribed to neutrino oscillations. Recent results from Kamiokande on higher energy neutrinos strengthen the case for an atmospheric neutrino problem. The relevant oscillation parameters were discussed here by Inoue 72 .

\section{RECONCILING PRESENT HINTS}

\subsection{Almost Degenerate Neutrinos}

It is not easy to account for the three observations cosmology and astrophysics discussed above in a framework containing just the three known neutrinos. The only possibility to fit these observations in a three-neutrino world is if all of them have nearly the same mass $\sim 2$ $\mathrm{eV}$ [73. This can be arranged, for example in general seesaw models which also contain an effective triplet vacuum expectation value [74, 1 , contributing to the light neutrino masses. This term should be added to eq. (2). Thus one can construct extended seesaw models where the main contribution to the light neutrino masses $(\sim 2 \mathrm{eV})$ is universal, due to a suitable horizontal symmetry, while the splittings between $\nu_{e}$ and $\nu_{\mu}$ explain the solar neutrino deficit and that between $\nu_{\mu}$ and $\nu_{\tau}$ explain the atmospheric 


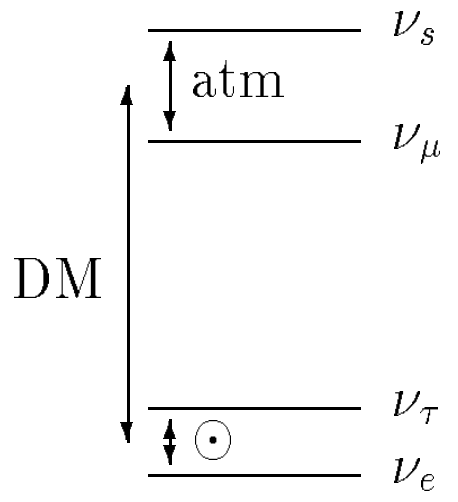

Figure 8. "Heavy" Sterile 4-Neutrino Model neutrino anomaly 75 . For a study of the required parameters, see ref. [76].

\subsection{Four-Neutrino Models}

The simplest way to fit all the data is to add a fourth neutrino species which, from the LEP data on the invisible $\mathrm{Z}$ width, we know must be of the sterile type, call it $\nu_{s}$. The first scheme of this type gives mass to only one of the three neutrinos at the tree level, keeping the other two massless 777. In a seesaw scheme with broken lepton number, radiative corrections involving gauge boson exchanges will give small masses to the other two neutrinos $\nu_{e}$ and $\nu_{\mu}$ [78. However, since the singlet neutrino is super-heavy in this case, there is no room to account for the three hints discussed above.

Two basic schemes have been suggested to keep the sterile neutrino light due to a special symmetry. In addition to the sterile neutrino $\nu_{s}$, they invoke additional Higgs bosons beyond that of the Standard Model, in order to generate radiatively the scales required for the solar and atmospheric neutrino conversions. In these models the $\nu_{s}$ either lies at the dark matter scale [79] as illustrated in Fig. 9 or, alternatively, at the solar neutrino scale 80]. In the first case the atmospheric neutrino puzzle is explained by $\nu_{\mu}$ to $\nu_{s}$ oscillations, while in the second it is explained by $\nu_{\mu}$ to $\nu_{\tau}$ oscillations. Correspondingly, the deficit of solar neutrinos is explained in the first case by $\nu_{e}$ to $\nu_{\tau}$ oscillations, while in the second

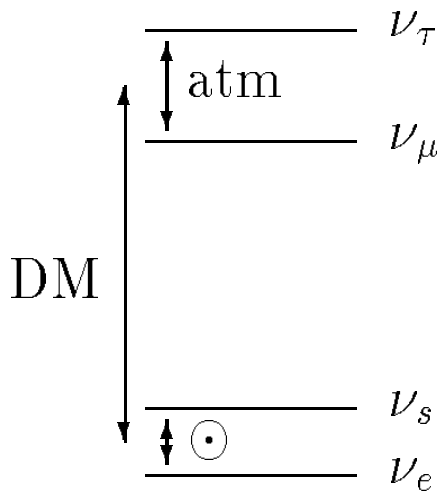

Figure 9. Light Sterile 4-Neutrino Model

it is explained by $\nu_{e}$ to $\nu_{s}$ oscillations. In both cases it is possible to fit all observations together. However, in the first case there is a clash with the bounds from big-bang nucleosynthesis. In the latter case the $\nu_{s}$ is at the MSW scale so that nucleosynthesis limits are satisfied. They nicely agree with the best fit points of the atmospheric neutrino parameters from Kamiokande [72]. Moreover, it can naturally fit the hints of neutrino oscillations of the LSND experiment [31]. For a more general study of the required parameters in four-neutrino models, see ref. [81].

Another theoretical possibility is that all active neutrinos are very light, while the sterile neutrino $\nu_{s}$ is the single neutrino responsible for the dark matter [82].

\subsection{Mev Tau Neutrino}

An $\mathrm{MeV}$ range tau neutrino is an interesting possibility to consider for two reasons. First, such mass is within the range of the detectability, for example at a tau-charm factory [27]. On the other hand, if such neutrino decays before the matter dominance epoch, its decay products would add energy to the radiation, thereby delaying the time at which the matter and radiation contributions to the energy density of the universe become equal. Such delay would allow one to reduce the density fluctuations on the smaller scales purely within the standard cold dark matter scenario, and could thus reconcile the large scale fluctua- 
tions observed by COBE 60 with the observations such as those of IRAS 61] on the fluctuations on smaller scales.

In ref. 83 a model was presented where an unstable MeV Majorana tau neutrino naturally reconciles the cosmological observations of large and small-scale density fluctuations with the cold dark matter model (CDM) and, simultaneously, with the data on solar and atmospheric neutrinos discussed above. The solar neutrino deficit is explained through long wavelength, so-called just-so oscillations 84 involving conversions of $\nu_{e}$ into both $\nu_{\mu}$ and a sterile species $\nu_{s}$, while the atmospheric neutrino data are explained through $\nu_{\mu} \rightarrow \nu_{e}$ conversions. Future long baseline neutrino oscillation experiments, as well as some reactor experiments will test this hypothesis. The model assumes the spontaneous violation of a global lepton number symmetry at the weak scale. The breaking of this symmetry generates the cosmologically required decay of the $\nu_{\tau}$ with lifetime $\tau_{\nu_{\tau}} \sim 10^{2}-10^{4}$ seconds, as well as the masses and oscillations of the three light neutrinos $\nu_{e}, \nu_{\mu}$ and $\nu_{s}$ required in order to account for the solar and atmospheric neutrino data. One can verify that the big-bang nucleosynthesis constraints 51,52 can be satisfied in this model.

\section{CONCLUSION}

Although theory alone can not predict neutrino masses, it is certainly true that neutrino masses are strongly suggested by present theoretical models of elementary particles. On the other hand, they seem to be required to account for present astrophysical and cosmological observations. Neutrino mass studies in nuclear $\beta$ decays and peak search experiments should continue. Searches for $\beta \beta_{0 \nu}$ decays with enriched germanium could test the quasi-degenerate neutrino scenario of section 5.1. Underground experiments at Superkamiokande, Borexino, and Sudbury will shed more light on the solar neutrino issue. Oscillation searches in the $\nu_{e} \rightarrow \nu_{\tau}$ and $\nu_{\mu} \rightarrow \nu_{\tau}$ channels at accelerators should soon improve over the present situation illustrated in
Fig. 2, while long-baseline experiments both at reactors and accelerators are being considered. The latter will test the regions of oscillation parameters presently suggested by atmospheric neutrino anomaly. Finally, new satellite experiments will test different models of structure formation, and shed light on the possible role of neutrinos as dark matter. If neutrinos are massive they could be responsible for a wide variety of implications, covering an impressive range of energies. These could be probed in experiments performed at underground installations as well as particle accelerators.

\section{REFERENCES}

1. For a review see A. Yu. Smirnov, plenary talk at 28th International Conference on Highenergy Physics (ICHEP 96), Warsaw, Poland, 25-31 Jul 1996, hep-ph/9611465.

2. For a review see J. W. F. Valle, Gauge Theories and the Physics of Neutrino Mass, Prog. Part. Nucl. Phys. 26 (1991) 91-171 (ed. A. Faessler)

3. M Gell-Mann, P Ramond, R. Slansky, in $S u-$ pergravity, ed. D. Freedman et al. (1979); T. Yanagida, in KEK lectures, ed. O. Sawada et al. (1979) R. Mohapatra and G. Senjanovic, Phys. Rev. Lett. 44 (1980) 912

4. R.N. Mohapatra and G. Senjanovic, Phys. Rev. D23 (1981) 165 and references therein.

5. D. Wyler, L. Wolfenstein, Nucl. Phys. B218 (1983) 205

6. R. Mohapatra, J. W. F. Valle, Phys. Rev. D34 (1986) 1642; J. W. F. Valle, Nucl. Phys. B (Proc. Suppl.) B11 (1989) 118-177

7. J. Bernabeu, A. Santamaria, J. Vidal, A. Mendez, J. W. F. Valle, Phys. Lett. B187 (1987) 303; J. G. Korner, A. Pilaftsis, K. Schilcher, Phys. Lett. B300 (1993) 381

8. G. C. Branco, M. N. Rebelo, J. W. F. Valle, Phys. Lett. B225 (1989) 385; N. Rius, J. W. F. Valle, Phys. Lett. B246 (1990) 249

9. M. Dittmar, M. C. Gonzalez-Garcia, A. Santamaria, J. W. F. Valle, Nucl. Phys. B332 (1990) 1; M. C. Gonzalez-Garcia, A. Santamaria, J. W. F. Valle, ibid. B342 (1990) 108.

10. J.W.F. Valle, Phys. Lett. B199 (1987) 432 
11. H. Nunokawa, Y.Z. Qian, A. Rossi, J.W.F. Valle, Phys. Rev. D54 (1996) 4356-4363, [ hep-ph/9605301

12. M. C. Gonzalez-Garcia, J. W. F. Valle, Mod. Phys. Lett. A7 (1992) 477; erratum Mod. Phys. Lett. A9 (1994) 2569; A. Ilakovac, A. Pilaftsis, Nucl. Phys. B437 (1995) 491; A. Pilaftsis, Mod. Phys. Lett. A9 (1994) 3595

13. L. Hall, V. Kostelecky, S. Raby, Nucl. Phys. B267 (1986) 415

14. F. Borzumati, A. Masiero, Phys. Rev. Lett. 57 (1986) 961; T. Kosmas, G. Leontaris, J. Vergados Phys. Lett. B219 (1989) 457; A. Gabbiani, A. Masiero, Nucl. Phys. B322 (1989) 235

15. R. Barbieri, L. Hall, A. Strumia, Nucl. Phys. B445 (1995) 219

16. M. Dittmar, J. W. F. Valle, contribution to the High Luminosity at LEP working group, yellow report CERN-91/02, p. 98-103

17. R. Alemany et. al. hep-ph/9307252, published in ECFA/93-151, ed. R. Aleksan, A. Ali, p. 191-211

18. Opal collaboration, Phys. Lett. B254 (1991) 293 and Zeit. fur Physik C67 (1995) 365; L3 collaboration, Phys. Rep. 236 (1993) 1146; Phys. Lett. B316 (1993) 427, Delphi collaboration, Phys. Lett. B359 (1995) 411.

19. M. C. Gonzalez-Garcia, J. W. F. Valle, Phys. Lett. B216 (1989) 360.

20. A. Zee, Phys. Lett. B93 (1980) 389; K. S. Babu, Phys. Lett. B203 (1988) 132

21. J. T. Peltoniemi, and J W F Valle, Phys. Lett. B304 (1993) 147;

22. Y. Chikashige, R. Mohapatra, R. Peccei, Phys. Rev. Lett. 45 (1980) 1926

23. A. Joshipura and J. W. F. Valle, Nucl. Phys. B397 (1993) 105 and references therein; J. C. Romao, F. de Campos, and J. W. F. Valle, Phys. Lett. B292 (1992) 329; A. S. Joshipura, S. Rindani, Phys. Rev. Lett. 69 (1992) 3269

24. Partcle Data Group, Phys. Rev. D54 (1996) 1-720 (see also URL: http://pdg.lbl.gov/).

25. K. Assamagan et al, Phys. Rev. D53 (1996) 6065

26. D. Buskulic et al., Phys. Lett. B349 (1995) 585; see also OPAL Collaboration, CERN-PPE-96-042, paper to submitted to the ICHEP 96 Conference, Warsaw, Poland, 25-31 Jul 1996.

27. J. Gómez-Cadenas, M. C. González-García, Phys. Rev. D39 (1989) 1370; J. GómezCadenas et al., Phys. Rev. D41 (1990) 2179; see also Third Workshop on the Charm Tau Factory, Marbella, Spain, June 1993, (World Scientific, 1994), Ed. J. Kirkby and R. Kirkby.

28. A. I. Belesev et al., Phys. Lett. B 350 (1995) 263; V. M. Lobashev, Talk given at the Int. Conf. Neutrino-96, Helsinki, June 1996.

29. B. Achkar et al. (BUGEY collabpration) Nucl. Phys. B434 (1995) 503; G.S. Vidyakin et al. (Krasnoyarsk), Pis'ma Zh. Eksp. Theor. Fiz. 59 (1994) 364; JETP 59 (1994) 390.

30. Karmen collaboration, B. Armbruster et al. Nucl. Phys. B (Proc. Suppl.) B38 (1995) 235.

31. C. Athanassopoulos, Phys. Rev. Lett. 75 (1995) 2650; J. E. Hill, Phys. Rev. Lett. 75 (1995) 2654; H. White, contribution to 1996 Warsaw conference.

32. E531 Collaboration, N. Ushida et al., Phys. Rev. Lett. 57 (1986) 2897.

33. E776 Collaboration, L. Borodovsky et al., Phys. Rev. Lett. 68 (1992) 274.

34. J. Gómez-Cadenas, M. C. González-García, Zeit. fur Physik C71 (1996) 443-454, hep$\mathrm{ph} / 9504246$

35. CHORUS and NOMAD proposals CERNSPSLC/91-21 (1992) and CERN-SPSC/90-42 (1992); J. Gómez-Cadenas, J. A. Hernando, A. Bueno, CERN-PPE/95-177; K. Kodama et al., FNAL proposal P803 (1991)

36. M. Tanimoto, Phys. Rev. D55 (1997) 322; J. Arafune, J. Sato, hep-ph/9607437

37. H. Klapdor, Prog. Part. Nucl. Phys. 32 (1994) 261.

38. W. Haxton, Nucl. Phys. B (Proc. Suppl.) 31 (1993) 88.

39. L. Wolfenstein, Nucl. Phys. B186 (1981) 147; J. W. F. Valle, Phys. Rev. D27 (1983) 1672 and references therein

40. J. Schechter and J. W. F. Valle, Phys. Rev. D25 (1982) 2951

41. E. Kolb, M. Turner, The Early Universe, Addison-Wesley, 1990, and references therein 42. J. Bardeen, J. Bond and G. Efstathiou, As- 
trophys. J. 321 (1987) 28; J. Bond and G. Efstathiou, Phys. Lett. B265 (1991) 245; M. Davis et al., Nature 356 (1992) 489; S. Dodelson, G. Gyuk and M. Turner, Phys. Rev. Lett. 72 (1994) 3754; H. Kikuchi and E. Ma, Phys. Rev. D51 (1995) 296; H. B. Kim and J. E. Kim, Nucl. Phys. B433 (1995) 421; M. White, G. Gelmini and J. Silk, Phys. Rev. D51 (1995) 2669 A. S. Joshipura and J. W. F. Valle, Nucl. Phys. B440 (1995) 647.

43. J. Schechter, J. W. F. Valle, Phys. Rev. D25 (1982) 774

44. J. W. F. Valle, Phys. Lett. B131 (1983) 87; G. Gelmini, J. W. F. Valle, Phys. Lett. B142 (1984) 181; J. W. F. Valle, Phys. Lett. B159 (1985) 49; M. C. Gonzalez-Garcia, J. W. F. Valle, Phys. Lett. B216 (1989) 360. A. Joshipura, S. Rindani, Phys. Rev. D46 (1992) 3000

45. J. C. Romão and J. W. F. Valle. Phys. Lett. B272 (1991) 436; Nucl. Phys. B381 (1992) 87.

46. R.F. Carswell, M. Rauch, R.J. Weynman et al., MNRAS 268 (1994) L1; A. Songalia, L.L. Cowie, C. Hogan and M. Rugers, Nature $\mathbf{3 6 8}$ (1994) 599.

47. D. Tytler and X.M. Fan, Bull. Am. Astr. Soc. 26 (1994) 1424.

48. N. Hata et al., Phys. Rev. Lett. 75 (1995) 3977.

49. C.J. Copi, D.N. Schramm and M.S. Turner, Science 267 (1995) 192 and Phys. Rev. Lett. 75 (1995) 3981; K. A. Olive and G. Steigman, Phys. Lett. B354 (1995) 357-362.

50. S. Sarkar, Reports on Progress in Physics 59 (1996) 1; P. J. Kernan and S. Sarkar, Phys. Rev. D 54 (1996) R3681

51. E. W. Kolb, M. S. Turner, A. Chakravorty and D. N. Schramm, Phys. Rev. Lett. 67 (1991) 533.

52. A.D. Dolgov and I.Z. Rothstein, Phys. Rev. Lett. 71 (1993) 476.

53. M. Kawasaki, G. Steigman and H.-S. Kang, Nucl. Phys. B402 (1993) 323, Nucl. Phys. B419 (1994) 105; S. Dodelson, G. Gyuk and M.S. Turner, Phys. Rev. D49 (1994) 5068; for a review see G. Steigman; in Cosmological Dark Matter, (World Scientific, 1994), ed. A.
Perez, and J. W. F. Valle, p. 55

54. A.D. Dolgov, S. Pastor, J.C. Romão and J.W.F. Valle, hep-ph/9610507

55. A Masiero, J. W. F. Valle, Phys. Lett. B251 (1990) 273 ; J. C. Romão, C. A. Santos, and J. W. F. Valle, Phys. Lett. B288 (1992) 311

56. G. Raffelt, Stars as laboratory for fundamental physics, Univ of Chicago press, ChicagoLondon (1996).

57. Y.-Z. Qian, G. M. Fuller, G. J. Mathwes, R. W. Mayle, J. R. Wilson and S. E. Woosley, Phys. Rev. Lett. 71 (191965) 93.

58. A. Yu. Smirnov, D. N. Spergel and J. N. Bahcall, Phys. Rev. D49 (1994) 1389.

59. E.L. Wright et al., Astrophys. J. 396 (1992) L13; M. Davis, F.J. Summers, and D. Schagel, Nature 359 (1992) 393; A.N. Taylor and M. Rowan-Robinson, ibid. 359 (1992) 396; R.K. Schaefer and Q. Shafi, Nature 359 (1992) 199; J.A. Holtzman and J.R. Primack, Astrophys. J. 405 (1993) 428; A. Klypin et al., Astrophys. J. 416 (1993) 1 J.A. Holtzman et al. Phys. Rev. Lett. 74 (1995) 2160.

60. G. F. Smoot et al., Astrophys. J. 396 (1992) L1-L5; E.L. Wright et al., Astrophys. J. 396 (1992) L13

61. R. Rowan-Robinson, in Cosmological Dark Matter, (World Scientific, 1994), ed. A. Perez, and J. W. F. Valle, p. 7-18

62. B.T. Cleveland et al., Nucl. Phys. B (Proc. Suppl.) 38 (1995) 47.

63. Y. Suzuki, Nucl. Phys. B (Proc. Suppl.) B38 (1995) 54

64. GALLEX Collaboration, P. Anselmann et al., LNGS Report 95/37 (June 1995).

65. SAGE Collaboration, J.S. Nico et al., Proc. 27th Conf. on High Energy Physics, Glasgow, UK (July 1994).

66. J. N. Bahcall and R. K. Ulrich, Rev. Mod. Phys. 60 (1990) 297; J. N. Bahcall and M. H. Pinsonneault, Rev. Mod. Phys. 64 (1992) 885 and Rev. Mod. Phys. 67 (1995) 781.

67. J. N. Bahcall, Phys. Lett. B338 (1994) 276; V. Castellani, et al Phys. Lett. B324 (1994) 245 ; N. Hata, S. Bludman, and P. Langacker, Phys. Rev. D49 (1994) 3622; V. Berezinsky, Comments on Nuclear and Particle Physics 21 (1994) 249 
68. M. Mikheyev, A. Smirnov, Sov. J. Nucl. Phys. 42 (1986) 913; L. Wolfenstein, Phys. Rev. D17 (1978) 2369; ibid. D20 (1979) 2634.

69. A.B. Balantekin, J.M. Fetter and F.N. Loreti, Phys. Rev. D54 (1996) 3941-3951

70. H. Nunokawa, A. Rossi, V. Semikoz, J. W. F. Valle, Nucl. Phys. B472 (1996) 495-517 [see also talk at 17th International Conference on Neutrino Physics and Astrophysics (Neutrino 96), hep-ph/9610526; C.P. Burgess, D. Michaud talk at Neutrino 96, hep-ph/9611368; E. Torrente-Lujan, hep$\mathrm{ph} / 9602398$

71. T. Stanev, Nucl. Phys. B (Proc. Suppl.) 48 (1996) 165; B. Barish, in proceedings of Int. Workshop on Elementary Particle Physics: Present and Future, ed. A. Ferrer and J. W. F. Valle, World Scientific, ISBN 981-02-25547.

72. K. Inoue, these proceedings

73. D.O. Caldwell and R.N. Mohapatra, Phys. Rev. D48 (1993) 3259; A. S. Joshipura, Zeit. fur Physik C64 (1994) 31; S. T. Petcov, A. Smirnov, Phys. Lett. B322 (1994) 109

74. J. Schechter and J. W. F. Valle, Phys. Rev. D22 (1980) 2227

75. A. Ioannissyan, J.W.F. Valle, Phys. Lett. B332 (1994) 93-99; B. Bamert, C.P. Burgess, Phys. Lett. B329 (1994) 289; D. Caldwell and R. N. Mohapatra, Phys. Rev. D50 (1994) 3477; D. G. Lee and R. N. Mohapatra, Phys. Lett. B329 (1994) 463; A. S. Joshipura, Phys. Rev. D51 (1995) 1321

76. H. Minakata, hep-ph/9612259

77. J. Schechter and J. W. F. Valle, Phys. Rev. D21 (1980) 309

78. See, for example, D. Choudhury et al Phys. Rev. D50 (1994) 3486

79. J. T. Peltoniemi, D. Tommasini, and J W F Valle, Phys. Lett. B298 (1993) 383

80. J. T. Peltoniemi, and J W F Valle, Nucl. Phys. B406 (1993) 409; E. Akhmedov, Z. Berezhiani, G. Senjanovic and Z. Tao, Phys. Rev. D47 (1993) 3245.

81. N. Okada, and O. Yasuda, hep-ph/9606411

82. J. T. Peltoniemi, Mod. Phys. Lett. A38 (1993) 3593

83. A. S. Joshipura, J. W. F. Valle, Nucl. Phys.
B440 (1995) 647.

84. For recent references see B. Faid, G.L. Fogli, E. Lisi, D. Montanino, Phys. Rev. D55 (1997) 1353-1364; Z. G. Berezhiani, A. Rossi, Phys. Lett. B367 (1996) 219-225; E. Calabresu, N. Ferrari, G. Fiorentini, M. Lissia, Astropart. Phys. 4 (1995) 159-176 hep-ph 9507352] 